\title{
OTIONOMTCS
}

Revista de los Estudios de Economía y Empresa

Dossier «Redes sociales, economía y empresa»

LOS PROCESOS DE TRANSMISIÓN DE INFORMACIÓN

\section{Organizaciones, redes sociales y gestión del conocimiento}

\section{Agustí Canals}

Profesor de los Estudios de Ciencias de la Información y la Comunicación (UOC)

RESUMEN Las redes sociales juegan un papel muy relevante como sustrato, sobre el que se producen la mayoría de los procesos relacionados con la transmisión de información y la generación y transferencia de conocimiento. La gestión del conocimiento, que tiene como una de sus funciones lograr optimizar estos procesos en las organizaciones, debe tener en cuenta esta función esencial de las redes sociales. En este artículo describimos algunos de los mecanismos concretos por los que la estructura de las redes sociales influye en la efectividad de la transmisión de información y también de la creación y transferencia de conocimiento. A partir de esto, se pueden deducir algunas consideraciones sobre las características de las redes sociales que son más indicadas en cada caso para la consecución de los objetivos de la organización. La gestión del conocimiento debe saber utilizar las redes existentes y, si es posible, conseguir que estas redes adopten la estructura más adecuada.

PALABRAS CLAVE redes sociales; gestión del conocimiento; organización; gestión de información

\section{Organisations, Social Media, and Knowledge Management}

\begin{abstract}
Social media play a relevant role as the basis to most processes related to information transfer as well as knowledge creation and transfer. In order to optimise those processes in organisations, knowledge management needs to take into account essential function of social media. In this article we describe some of the specific mechanisms by which a social media structure influences the effectiveness of information transfer and knowledge creation and transfer. Based on this, we put forward some considerations on the most suitable features of social media to meet organisational objectives. Knowledge management must be able to use existing social media and, if possible, to have those media adopt the most adequate structure.
\end{abstract}

KEYWORDS social media; knowledge management; organisation, information management

\section{Introducción}

Cuando escuchamos el término redes sociales, normalmente pensamos en el conjunto de personas interrelacionadas a través de las plataformas en línea llamadas "medios sociales", como son, por citar los más populares, Facebook o Twitter. Pero no son necesarios los medios sociales, ni tan solo Internet, para que existan las redes 
sociales. De hecho, las redes sociales existen desde el momento en que los humanos (o sus antepasados) empezaron a vivir en comunidad. Cualquier grupo de personas entre las cuales se establezca algún tipo de relaciones que las enlacen entre ellas ya forma una red social. Incluso podemos decir que también forman redes sociales las interrelaciones entre familias, empresas, ciudades o países.

Es evidente, pues, que las redes sociales están presentes en toda la sociedad, y en particular en las organizaciones, tanto en las empresas privadas como en las instituciones públicas y en el tercer sector. Los miembros de las organizaciones forman redes sociales entre ellos y con personas de fuera de la organización, y las organizaciones como tales forman redes sociales con otras organizaciones. Conocer cómo se forman estas redes y los efectos que tienen en los procesos que se llevan a cabo dentro de las organizaciones y entre ellas es importante. Nos permite entender mejor cómo funcionan las organizaciones y nos ayuda a gestionarlas mejor.

Uno de los aspectos en los que las redes sociales tienen un rol más relevante es la difusión de información, y como consecuencia, también en los procesos de creación y de transferencia de conocimiento. Así pues, si queremos gestionar bien la información y el conocimiento, debemos tener en cuenta las redes sociales. En este artículo veremos algunos de los aspectos de las redes sociales que son más relevantes a la hora de gestionar el conocimiento en las organizaciones. Comenzaremos repasando algunos conceptos básicos sobre la importancia de la información y el conocimiento en las organizaciones. A continuación veremos cómo las redes sociales juegan un papel muy importante en la transmisión de información y los efectos que puede tener esto en las organizaciones. Y para finalizar, examinaremos el efecto de las redes sociales en los procesos de creación y transferencia de conocimiento, que van más allá de la simple transmisión de información.

\section{Información y conocimiento en las organizaciones}

Una organización se puede definir, de forma sencilla, como una estructura social diseñada para hacer cosas a través de la cooperación de individuos (Kadushin, 2012). Podemos decir, pues, que una organización persigue una serie de objetivos, que pueden ser implícitos o explícitos, y los individuos que la forman se estructuran y actúan de forma conjunta con el fin de lograr estos objetivos. Tan solo a partir de esta definición, ya podemos ver que el papel tanto de la información como del conocimiento será muy relevante. Para poder actuar adecuadamente, se necesita conocimiento sobre el mundo que nos envuelve y sobre el efecto que pueden tener nuestras acciones. Y la información necesaria para poderse coordinar y para poder adquirir nuevo conocimiento.

Tanto el concepto de información como el de conocimiento tienen muchas facetas y esto hace que sean difíciles de definir de modo sencillo. A pesar de que el conocimiento ha sido una de las preocupaciones fundamentales del hombre desde el inicio de la filosofía (Nonaka y Takeuchi, 1995) y que la información ha resultado en los últimos tiempos uno de los elementos clave de diversas disciplinas científicas (Gleick, 2011), no se ha llegado todavía a una definición clara y universal. Y, seguramente por esto, tampoco hay un consenso sobre la relación entre información y conocimiento.

Sin querer entrar aquí en profundidad en este tema, sí que es necesario describir, a grandes rasgos, lo que entendemos por información y conocimiento. El conocimiento es eso que permite a un agente determinado interpretar y entender su entorno y actuar en consecuencia. Así, nuestro conocimiento sobre las normas de circulación hace que podamos prever (en teoría) el comportamiento de los otros conductores y conducir del modo adecuado para llegar a nuestro destino. La información, en cambio, es todo eso que el agente puede extraer de su entorno que le permite modificar su base de conocimiento. Por ejemplo, sabemos que el código de circulación permite ir a una velocidad máxima por la carretera. Ahora bien, si vemos una señal que limita la velocidad en una zona concreta, esta información nos hace modificar nuestro conocimiento: ahora ya sabemos que no podemos ir a $100 \mathrm{~km} / \mathrm{h}$, sino que tenemos que reducir a $60 \mathrm{~km} / \mathrm{h}$. Notemos que, mientras que el conocimiento es una propiedad del agente, la información es algo externo. El agente encuentra la información en su entorno, y también puede ser que actúe sobre el entorno de modo que otros agentes puedan encontrar información. Entonces se produce la transmisión de información y, como consecuencia, la transferencia de conocimiento. En nuestro ejemplo, las autoridades de tránsito ponen una señal en un tramo para que los conductores adquieran el conocimiento necesario para poder conducir con seguridad. Estas ideas están presentadas más en detalle en algún trabajo anterior (Boisot y Canals, 2004). 
El lector habrá notado que en el párrafo anterior nos hemos referido siempre al conocimiento del "agente" y no al del individuo o la persona. Esto es porque la definición de conocimiento es suficientemente general como para que pueda aplicarse a cualquier ente con capacidad de actuar (es decir, a cualquier agente). Así, las personas pueden tener evidentemente conocimiento, pero también los animales, los robots o los sistemas expertos. Y también podemos pensar que hay conocimiento en diversos niveles: cada uno de los miembros de una empresa tiene un conocimiento individual, pero la empresa en conjunto también tiene un conocimiento, porque es un agente capaz, por ejemplo, de interpretar el mercado y actuar del modo que sea más oportuno para sus intereses.

La gestión del conocimiento se ocupa de crear las condiciones para que el conocimiento de las organizaciones en conjunto se adecue al máximo al que necesita para cumplir sus objetivos. Esto quiere decir que se debe ocupar tanto del conocimiento organizativo en general como del conocimiento que cada uno de los miembros de la organización necesita para llevar a cabo sus tareas individuales. La gestión del conocimiento debe tener como objetivo optimizar los procesos de generación y transmisión del conocimiento, tanto los internos como los que involucren elementos externos a la organización. Y debe tener en cuenta tanto el conocimiento necesario para la operativa del día a día como el conocimiento de índole más estratégico. Si hemos dicho que una organización es una estructura social, no es nada extraño que en todos estos temas las redes sociales influyan, como veremos a continuación.

\section{El papel de las redes sociales}

En la mayoría de los procesos relacionados con la información y el conocimiento, las redes sociales juegan un papel muy importante. A continuación veremos que la transmisión de información se produce muchas veces a través de los "caminos" que configuran las redes sociales, y que tanto a la hora de generar conocimiento como de transferirlo de unos agentes a otros, las configuraciones de las redes sociales tienen efectos sobre la eficacia y la eficiencia del proceso.

\subsection{Transmisión de información}

Por la teoría de la información sabemos que la transmisión de información entre un emisor y un receptor se hace por un canal (Shannon, 1948). Normalmente, este canal se entiende como un medio por el cual circula la información, como el aire o los cables de teléfono. Pero la mayoría de veces, para que se transmita la información debe haber también lo que podríamos llamar un "canal social". Es decir, un vínculo entre emisor y receptor que facilite la comunicación. Puede ser que se conozcan previamente, puede ser que estén en el mismo sitio en el mismo momento, puede ser que compartan unos códigos culturales y lingüísticos... Si nos fijamos, vemos que este tipo de vínculos son los que configuran las redes sociales. Así pues, podríamos decir que la información "viaja" por las redes sociales. Por ejemplo, si un trabajador se percata de una buena oferta de trabajo en su empresa, lo hará saber a sus conocidos, a quien crea que le pueda interesar. No lo dirá a su vecino si no le conoce y no sabe que aquella oferta le podrá ir bien.

Ahora bien, para que haya transmisión de información también es necesario que el emisor y el receptor cumplan algunos requisitos. Por parte del emisor, normalmente es necesario que haya voluntad de emitir la información. Es verdad que a veces la información se emite involuntariamente, ya sea oralmente o por escrito, o a través de lenguaje no verbal. Pero lo más normal es que el emisor codifique voluntariamente un mensaje para hacerlo llegar a los posibles receptores. Estos receptores, por su parte, de entrada deben estar atentos al mensaje. Y esto no es tan fácil, especialmente cuando hay una gran sobrecarga de estímulos en el entorno que compiten por nuestra atención (Davenport y Beck, 2001). Además, es necesario que los receptores conozcan el código en el cual se ha emitido el mensaje y tengan suficiente conocimiento del contexto como para interpretarlo. Es lo que Shanno y Weaver (Shanno y Weaver, 1963) llaman, respectivamente, el problema semántico y el problema de efectividad.

Una de las condiciones previas para hacer una buena gestión del conocimiento es gestionar bien la información (Canals, 2003). Según lo que acabamos de decir, pues, será necesario que se tengan en consideración las 
redes sociales como soporte a través de las cuales circulan los flujos de información y las características de los actores que forman parte de la red desde un punto de vista informacional.

Desde un punto de vista de la red social vista en conjunto, un aspecto interesante es cómo se difunde la información. Es decir, cómo una información que se genera por primera vez en uno de los nodos de la red se va difundiendo por todos los otros. Suponiendo que la transmisión entre los nodos se produce normalmente porque cumple las condiciones que hemos visto anteriormente, la rapidez con la que la información se difunde en la red depende mucho de la estructura. En redes poco densas (con pocas conexiones) y con una distancia media entre nodos muy alta (lo que se conoce como camino medio) costará mucho más difundir una información que en una con camino medio bajo (lo que a menudo se llama un mundo pequeño, y que es una característica bastante frecuente en las redes sociales) (Barabási, 2002; Watts y Strogatz 1998). En el caso de que haya errores en la transmisión (como en el popular juego infantil del "teléfono" donde un mensaje que se va pasando de uno a otro, de boca a oreja, acaba siendo irreconocible), la transmisión puede ser mucho más lenta y es posible que no llegue a difundirse en toda la red.

Un concepto que se debe destacar en la difusión de información en las redes sociales es el de vínculos débiles (Granovetter, 1973). La idea es que la información que podemos recibir de las personas que tenemos cerca y con quienes tenemos una relación más estrecha es probable que no constituya una gran novedad para nosotros, ya que nos movemos en el mismo entorno. Podemos decir que es más difícil que sea información que nos permita aumentar o modificar mucho nuestro conocimiento. En cambio, la información que nos puede llegar de personas más alejadas, con quien podemos tener una relación menos estrecha (un enlace más "débil"), puede sernos mucho más útil porque viene de entornos con los que no tenemos mucho contacto.

En vista de todos estos mecanismos, está claro que los actores de la red que puedan acceder más rápidamente a los flujos de información y controlarlos tendrán una ventaja sobre los otros. Los nodos más centrales, por ejemplo, dado que la información tenderá a pasar por su posición, a menudo pueden acceder antes y, además, decidir si la transmiten y a quién lo hacen. Hay diversas medidas posibles de centralidad de los nodos de una red en las cuales no podemos entrar en profundidad aquí (ver Borgatti, 2005; Canals et al., 2012; Newman, 2010; Wasserman y Faust, 1994), pero la mayoría tienen importancia en la difusión de información, con matices ligeramente distintos.

En la figura 1 podemos ver dos tipos ideales bien diferentes de estructura de la red social de una organización. En la primera, que llamaremos estrella por la forma que tiene, hay un nodo central por el cual debe pasar por obligación toda la información que circula por la red. La segunda, en cambio, que tiene forma de diamante, está altamente cohesionada: todos los nodos están conectados entre sí. Evidentemente, son casos extremos que pocas veces se dan en el mundo real, pero que nos son útiles para discutir unos cuantos conceptos.

Figura 1. Dos modelos ideales de estructura de la red social en las organizaciones

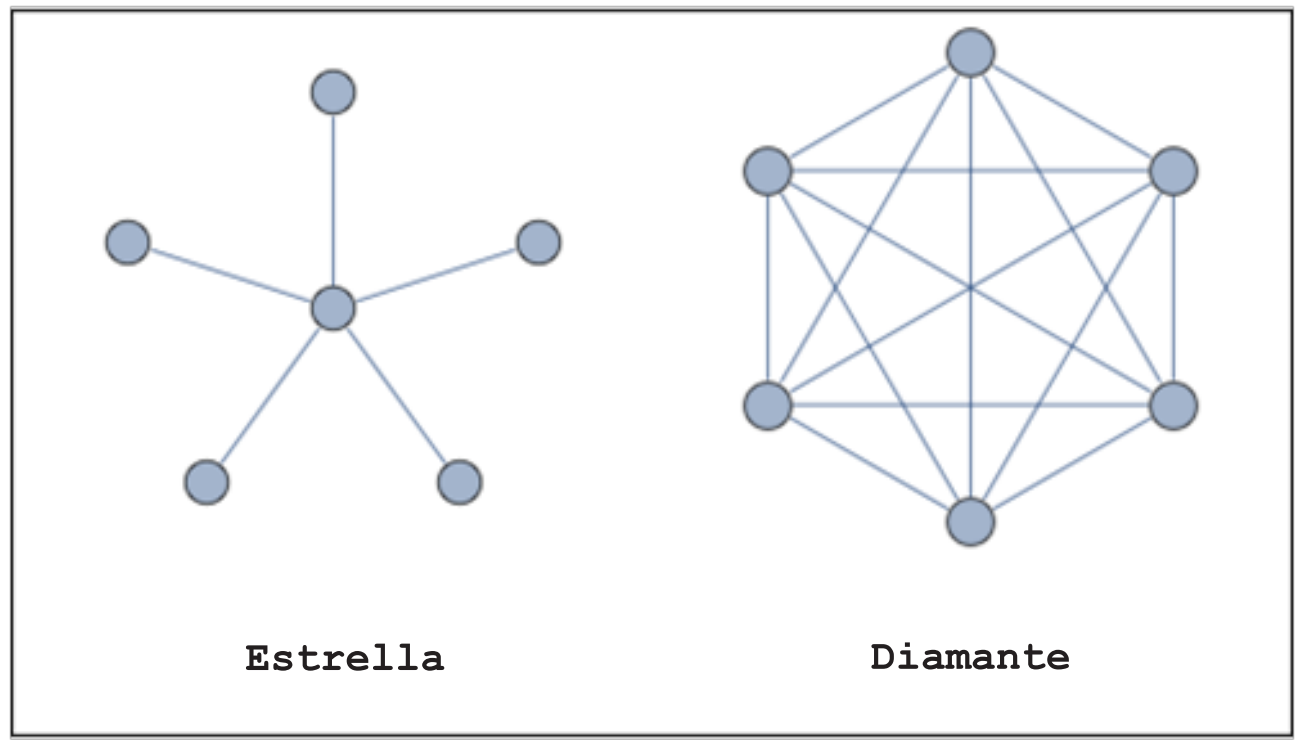


Una pregunta que nos podemos hacer es si para la organización será mejor una estructura de la red social de los miembros tipo estrella o tipo diamante. A partir de lo que hemos visto sobre la difusión de información en redes, parece claro que esta se difundirá mucho más fácilmente en la estructura tipo diamante. Cada nodo tiene muchas más vías de entrada y salida de información y todos los nodos tienen conexión directa entre sí, lo cual asegura una difusión rápida. Pero además, en la estructura tipo estrella toda la información debe pasar por un único nodo. Según la capacidad del nodo central para procesarla, puede convertirse en un cuello de botella y dificultar mucho la fluidez del proceso. Así pues, si a la empresa o institución le interesa fomentar la difusión de información, siempre es mejor una estructura tipo diamante, pero si necesita controlar los flujos de información, mejor que tenga una estructura de red social tipo estrella, siempre que el individuo central sea el adecuado. Se debe recordar que la estructura de la red social no tiene por qué coincidir (y a menudo es asi) con la estructura formal reflejada en el organigrama.

Ahora bien, desde el punto de vista de los individuos, la cosa cambia. Mientras que en la estructura diamante todos se encuentran en las mismas condiciones, en la estructura tipo estrella quien ocupa la posición central parece claro que tiene una situación privilegiada. Toda la información que transita por la red pasa por el nodo central, y él la conoce antes que nadie (a excepción del emisor). Si esto no le provoca problemas de sobrecarga, tiene una ventaja clara, ya que puede utilizar la información, e incluso tiene el poder de decidir qué información transmite y cuál no y a quién según sus intereses. Nuevamente, si sus intereses coinciden con los de la organización, no es un problema, pero esto no siempre es así.

Situaciones como la que se produce en la estructura tipo estrella con el nodo central reciben el nombre de agujeros estructurales (Burt, 1992). La idea es que un elemento de la red que conecta con dos nodos o grupos de nodos que no están conectados por ninguna otra vía aprovecha (o "llena") un agujero estructural, cosa que le confiere el control sobre la comunicación entre las dos partes y, por tanto, un grado de poder considerable.

\subsection{Generación y transferencia de conocimiento}

Las ideas anteriores sobre la transmisión de información en las redes son importantes a la hora de analizar los procesos relacionados con el conocimiento, ya que tal y como hemos visto se debe transmitir información para transferir conocimiento y también se necesita información para generar de nuevo. Pero se deberán tener en consideración otras cosas. Tal y como proponen algunas teorías sobre el capital social (Inkpen y Tsang, 2005; Nahapiet y Ghoshal, 1998), las redes sociales se pueden utilizar para tener acceso a otros recursos además de la información, como es la influencia, control o solidaridad social (Sandefur y Laumann, 1998). Y estos otros recursos también juegan un papel en la generación y transferencia de conocimiento.

Por ejemplo, no siempre que un actor recibe una información que podría hacer cambiar su base de conocimiento realmente pasa esto. Puede suceder que, si el actor no se fía de quien le ha enviado la información o bien no lo conoce, no crea que lo que le llega sea suficiente para cambiar sus creencias sobre el entorno, su conocimiento. Hay transmisión de información pero no transferencia de conocimiento. En algunos casos, los actores solo cambian su conocimiento cuando la misma información les llega de forma independiente de un porcentaje de sus vecinos en la red. Por ejemplo, en la figura 2 vemos una secuencia temporal en la que solo hasta que tres de los cinco vecinos del nodo central emiten una información (es decir, se ponen de color gris claro), este nodo no cambia su conocimiento volviéndose del mismo color. También puede haber transmisión de información pero no de conocimiento cuando el actor que la recibe no puede interpretarla porque no domina el código en el que se ha enviado el mensaje, o bien porque no tiene una base de conocimiento previa suficiente para interpretarla. 
Figura 2. Difusión de conocimiento según un umbral. El nodo central incorpora la información en su base de conocimiento después de que 3 de sus 5 vecinos lo han hecho, ya que tiene un umbral del $50 \%$.

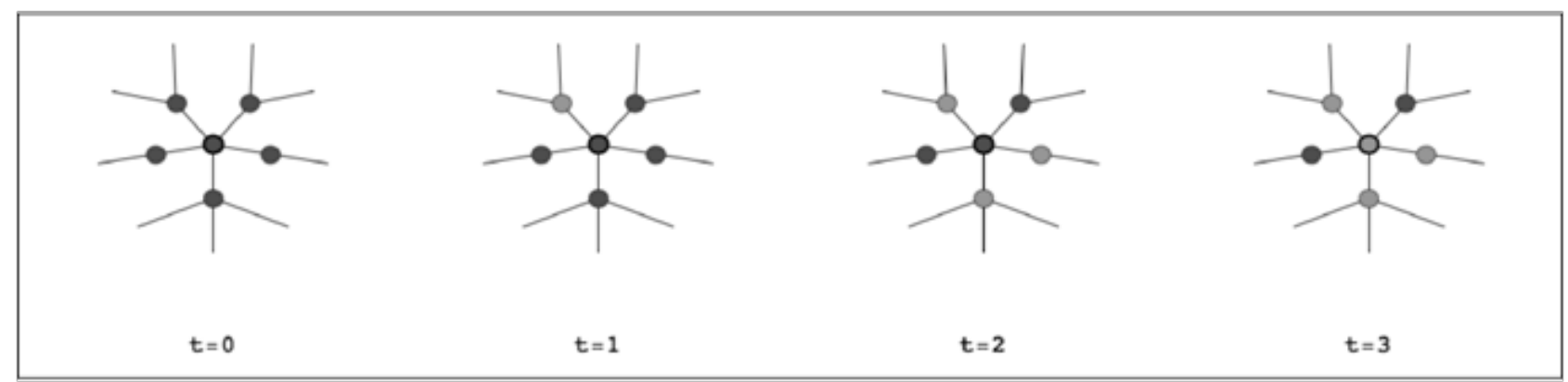

También la generación de conocimiento en las organizaciones es un proceso complejo. A menudo requiere de la acción conjunta de grupos de individuos que puedan compartir fácilmente información y también transferirse entre ellos conocimiento tácito. Por eso, en organizaciones que basan su actividad en la generación de nuevo conocimiento como las empresas innovadoras, las estructuras de tipo diamante pueden ser, no solo mejores, sino imprescindibles. Incluso desde el punto de vista individual del individuo central de una estructura inicialmente de tipo estrella, puede ser beneficioso a la larga renunciar a la posición privilegiada que tiene para ir hacia una estructura más de tipo diamante que favorezca la creación de conocimiento, de la cual se puede beneficiar todo el mundo.

En la literatura sobre aprendizaje organizacional, se hace a menudo la distinción entre la exploración de nuevas posibilidades y la explotación de lo que ya se conoce (March, 1991). Ambas son necesarias pero es muy difícil conseguir que las estructuras de una organización se adapten bien a las dos. A menudo lo que va bien para la exploración no va bien para la explotación. En el caso de las redes sociales, una estructura tipo estrella puede tener todo el sentido, porque lo que interesa es optimizar la eficiencia de los procesos conocidos. Y esto se puede hacer bien en una estructura jerárquica y burocratizada. En cambio, para la exploración, que requiere la generación de nuevo conocimiento, las estructuras tipo diamante parecen las más adecuadas. Las organizaciones que mejor balancean las dos actividades lo hacen sobre la base de la convivencia de dos tipos de estructuras. Cada parte de la organización adopta la que va mejor a su actividad de explotación o de exploración. Es probable, pues, que en la red social de muchas organizaciones haya partes que tienden más a estructura estrella y otras a estructura diamante.

Un ejemplo muy estudiado de grupos que necesitan una alta capacidad de aprendizaje y de generar conocimiento son las llamadas comunidades de práctica (Wenger, 1998). En las comunidades de práctica que funcionan se suele establecer una red social muy densa (tipo diamante) con vínculos fuertes en los que la transmisión de conocimiento, especialmente conocimiento tácito, es más fácil. No hay suficiente con trasmitirse información.

A pesar de las ventajas que hemos visto para la generación de conocimiento, una estructura cohesiva también puede tener algún inconveniente. Cuando un grupo está muy cohesionado, la densidad de cohesión con los miembros del grupo hace que se pueda reducir mucho la conexión con el exterior. Esto puede dificultar que entren ideas nuevas, que son esenciales para la creación de nuevo conocimiento. Por eso son importantes los enlaces débiles que hemos mencionado anteriormente. Tampoco es buena una estructura muy cohesionada tipo estrella cuando lo que se pretende es aprovechar los agujeros estructurales de la red. Por ejemplo, una agencia de head hunting lo que hace es precisamente poner en contacto empresas que necesiten cubrir puestos de trabajo con profesionales que lo pueden hacer. Si hay otros vínculos entre las empresas y los profesionales, como es el caso de portales online, el valor del servicio de la agencia disminuye mucho.

Diversos estudios sobre los flujos de conocimiento en organizaciones innovadoras, como las que se sitúan en clústeres geográficos especializados en nuevas tecnologías (Canals et al., 2008; Saxenian, 1994), confirman que este necesario ingrediente de conocimiento nuevo proveniente del exterior suele venir de enlaces débiles en las redes sociales personales de los trabajadores. Asimismo, este tipo de aportaciones también lo puede fomentar 
la organización de manera más o menos formalizada. Esta es la intención que hay detrás del establecimiento de algunas alianzas empresariales y de la presencia de altos cargos de algunas empresas en los consejos de administración de otras y que da lugar al que se conoce como consejerías cruzadas. Los dos fenómenos se pueden estudiar mediante el análisis de las redes sociales que producen. En el primer caso, son redes donde los nodos son organizaciones que están conectadas si tienen algún tipo de alianza, y en el otro, redes bipartidas donde hay unos nodos correspondientes a las empresas y los otros a los directivos. Un directivo estará conectado a las empresas en las que participa como miembro del consejo de administración.

En los últimos años las TIC han facilitado que empresas e instituciones puedan establecer formas de coordinación con otras organizaciones que ya no siguen el patrón tradicional de acuerdos contractuales entre estructuras burocráticas, sino que tienen un carácter más de sistema social informal en el que la confianza, la toma de decisiones distribuida y la negociación constante son elementos fundamentales. Esto ha dado lugar a sistemas relativamente estables de actores autónomos pero interdependientes que no son ni organizaciones jerárquicas ni mercados, y se llaman organizaciones red. Este tipo de organizaciones han demostrado que pueden ser más eficientes que las tradicionales en la adquisición de conocimiento y en la resolución de problemas gracias a su actuación descentralizada, y también más eficaces en la resolución de problemas porque pueden abordar y encontrar soluciones de forma colectiva.

\section{Conclusiones}

La importancia de las redes sociales como sustrato sobre el que se producen los procesos de transmisión de información y de generación y transferencia de conocimiento hace que sea inevitable considerarlas en toda aproximación a la gestión del conocimiento en las organizaciones. Pero debe hacerse teniendo en cuenta que no se pueden controlar nunca del todo. Es cierto que se pueden crear redes formales a partir de los organigramas. Y se puede mirar de fomentar redes informales mediante, por ejemplo, el diseño arquitectónico, la formación de equipos de trabajo, la definición de espacios apropiados como las cafeterías o la utilización de medios sociales en línea. Pero las redes sociales son sistemas complejos (Amaral y Ottino, 2004; Newman, 2010), y por tanto presentan propiedades de autoorganización que no permiten dominarlas como si fuesen sistemas mecánicos (Solé, 2009). La gestión del conocimiento debe ser capaz de detectar las redes existentes para aprovecharlas y fomentar la generación de vínculos que hagan evolucionarlas hacia la estructura que sea más conveniente para que la creación y la transferencia de conocimiento contribuyan a la consecución de los objetivos de la organización.

\section{Agradecimientos}

El autor agradece la ayuda financiera de la Generalitat de Catalunya a través del grupo de investigación KIMO (2014-SGR-1486) y del MINECO a través de la financiación del proyecto KIBIS (CSO2012-33959).

\section{Bibliografia}

AMARAL, L. A. N.; OTTINO, J. M. (2004). "Complex networks: Augmenting the framework for the study of complex systems". The European Physical Journal B. N. ${ }^{\circ}$ 38, págs. 147-162.

BARABÁSI, A.-L. (2002). Linked: The New Science of Networks. Edition ed. Cambridge, MA: Perseus. ISBN 0-7382-0667-9.

BOISOT, M. H.; CANALS, A. (2004). "Data, information and knowledge: have we got it right?". Journal of Evolutionary Economics. N. ${ }^{\circ}$ 14, págs. 43-67.

BORGATTI, S. P. (2005). "Centrality and network flow". Social Networks. N. 27, págs. 55-71.

BURT, R. S. (1992). Structural Holes: The Social Structure of Competition. Edition ed. Cambridge, MA: Harvard University Press. ISBN 0-674-84371-1.

CANALS, A. (2003). Gestión del conocimiento. Edition ed. Barcelona: Gestión 2000. ISBN 84-8088-844-X. 
CANALS, A.; BOISOT, M. H.; MACMILLAN, I (2008). "The spatial dimension of knowledge flows: a simulation approach". Cambridge Journal of Regions, Economy and Society. Vol. 2, n. ${ }^{\circ}$ 1, págs. 175-204.

CANALS, A.; DIAZ-GUILERA, A.; MOLINA, J. L.; PATRACA, B. (2012). Xarxes socials: Fonaments $i$ aplicacions. Edition ed. Barcelona: Editorial UOC.

DAVENPORT, T. H.; BECK, J. C. (2001). The attention economy. Edition ed. Boston, MA.: Harvard Business School Press.

GLEICK, J. (2011). The information: A history, a theory, a flood. Edition ed. London, UK: HarperCollins.

GRANOVETTER, M. (1973). "The strength of weak ties". American Journal of Sociology. Vol. 6, n. 78, págs. 1360-1380.

INKPEN, A. C.; TSANG, E. W. K. (2005). "Social capital, networks, and knowledge transfer". Academy of Management Review. Vol. 1, n. ${ }^{\circ}$ 30, págs. 146-165.

KADUSHIN, C. (2012). Understanding social networks: Theories, Concepts, and Findings. Edition ed. New York: Oxford University Press.

MARCH, J. G. (1991). "Exploration and Exploitation in Organizational Learning". Organization Science. Vol. 1, n. ${ }^{\circ}$ 2, págs. 71-87.

NAHAPIET, J.; GHOSHAL, S. (1998). Social Capital, Intellectual Capital, and the Organizational Advantage. Academy of Management Review. Vol. 2, n. ${ }^{\circ}$ 23, págs. 242-266.

NEWMAN, M. E. J. (2010). Networks: An Introduction. Edition ed. Oxford, UK: Oxford University Press.

NONAKA, I.; TAKEUCHI, H. (1995). The knowledge-creating company. Edition ed. New York: Oxford University Press. ISBN 0195092694.

SANDEFUR, R. L.; LAUMANN, E. O. (1998) "A paradigm for social capital”. Rationality and Society. Nov. 1, vol. 4, n. ${ }^{\circ}$ 10, págs. 481-501.

SAXENIAN, A. (1994). "Silicon Valley versus Route 128". En: Inc.com.

SHANNON, C. E. (1948). "The Mathematical Theory of Communication". Bell System Technical Journal. N. ${ }^{2} 27$, págs. 379-423, 623-656.

SHANNON, C. E.; WEAVER W. (1963). The mathematical theory of communication. Edition ed. Urbana: University of Illinois Press. ISBN 0-252-72548-4.

SOLÉ, R. V. (2009). Xarxes complexes: Del genoma a Internet. Edition ed. Barcelona: Empúries.

WASSERMAN, S.; FAUST, K. (1994). Social Network Analysis: Methods and Applications. Edition ed. Cambridge, UK.: Cambridge University Press.

WATTS, D. J.; STROGATZ, S. (1998). "Collective Dynamics of 'small-world' networks”. Nature. N. 393, págs. 440-442.

WENGER, E. (1998). Communities of practice: learning, meaning and identity. Edition ed. Cambridge, UK: Cambridge University Press. ISBN 0521663636.

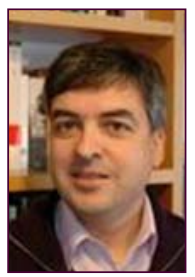

\section{Agustí Ganals \\ acanalsp@uoc.edu \\ Universitat Oberta de Catalunya \\ Profesor de los Estudios de Ciencias de la Información y la Comunicación (UOC)}

Licenciado y máster en Ciencias Físicas por la Universidad Autónoma de Barcelona, y MBA y doctor en Management Sciences por ESADE (Universidad Ramon Llull). Ha realizado estudios de posgrado en las universidades norteamericanas de Penn State y Syracurse y ha sido investigador visitante en la Warwick business School de la Universidad de Warwick, en la Wharton School de la Universidad de Pennsylvania y en la Saïd Business School de la Universidad de Oxford. Durante su vida profesional ha compaginado la actividad académica con la gestión empresarial y la consultoría en los ámbitos de organización, sistemas de información y gestión del conocimiento. 
Su investigación se centra en temas relacionados con la gestión estratégica de la información y el conocimiento, las redes sociales y la complejidad en las organizaciones. Actualmente es profesor agregado de la Universitat Oberta de Catalunya, donde fundó los Estudios de Ciencia de la Información y de la Comunicación, y también profesor asociado del Departamento de Dirección General y Estrategia en ESADE. Es también director del grupo de investigación consolidado KIMO sobre gestión de la información y el conocimiento en las organizaciones, y del máster universitario de Gestión estratégica de la información y el conocimiento en las organizaciones, de la UOC. Ha publicado diversos artículos especializados en revistas internacionales y los libros Gestión del Conocimiento (Ediciones 2000, 2003) y Xarxes socials: Fonaments i aplicacions (Editorial UOC, 2012).

Los textos publicados en esta revista están -si no se indica lo contrario- bajo una licencia Reconocimiento-Sin obras derivadas 3.0 España de Creative Commons. Puede copiarlos, distribuirlos y comunicarlos públicamente siempre que cite su autor y la revista y la institución que los publica (autoría, nombre de la revista, institución editora); no haga con ellos obras derivadas. La licencia completa se puede consultar en http://creativecommons.org/licenses/by-nd/3.0/es/deed.es.

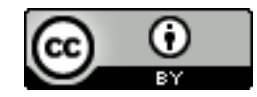

